Textures and Microstructures, 1988, Vols. 8 \& 9, pp. 131-151

Reprints available directly from the publisher

Photocopying permitted by license only

(C) 1988 Gordon and Breach Science Publishers Inc.

Printed in the United Kingdom

\title{
Description and Presentation Methods for Textures
}

\author{
JÜRGEN HIRSCH and KURT LÜCKE \\ Institute f. Allgemeine Metallkunde und Metallphysik, RWTH Aachen, W. \\ Germany
}

(Received July 6, 1987; in final form December 2, 1987)

\section{Dedicated to the memory of Professor Günter Wassermann}

Different methods to describe and analyze the peak and/or fibre characteristics of textures are discussed. In metallic materials the main features of a certain type of texture can clearly and comprehensively be described by using adequate extractions from the three-dimensional orientation distribution function (ODF), which is usually calculated from two-dimensional X-ray pole figures. The ODF has a higher resolving power, but the abstract form of orientation density data plotted in a threedimensional orientation space is not always the most suitable way for presentation and further interpretation. Methods to describe the textures with the help of ideal orientations and/or fibres, their quantitative analysis with the help of Gauss-type scattering components, the selective projection and plot of characteristic orientation fibres are discussed and compared. As an example the development of rolling textures of fcc metals is described and analyzed. Using these evaluation methods it can be demonstrated how their characteristic features are related to theoretical predictions and to microstructural effects.

KEY WORDS: ODF-analysis, model functions, peak textures, fibre textures, selective projection, fcc-bcc metals, rolling textures, recrystallization textures.

\section{INTRODUCTION}

Texture investigations have become more and more attractive during the last years for both fundamental research and industrial application and many scientists are involved with the mathematical techniques used to evaluate and describe textures (Bunge, 1987). 
With the help of big advances in measuring techniques and evaluation methods today very detailed data about the orientation distribution of the grains in a material are available. Additional to the usual metallographic aspects, like crystal structure, composition, size and shape of the grains, texture investigation is concerned with another fundamental attribute of grains, namely with their orientations and therefore it can be considered as an extension of metallography. Also the grain orientations strongly influence the properties of a material and, moreover, the investigation of their correlation to microstructure allows further interpretation of its determinative processes.

Like the grain sizes, also the orientations are usually considered in the form of statistical distributions, i.e. the volume fractions of the material containing grains of a certain diameter or orientation, respectively. Hence a homogeneous material with equiaxed grains would be described by the distribution function $F(r, g)$, where $r$ is the grain radius and $g$ is the orientation, (e.g. given by the three Euler angles $\varphi_{1}, \Phi, \varphi_{2}$ ) (Bunge, 1969) and $F d r d g$ represents the volume fraction. So far, however, not the combined distribution function $F(r, g)$, but the size distribution function ("SDF") $\varphi(r)$ and the orientation distribution function ("ODF") $f(g)$ are determined separately. The topological arrangement of the different types of grains is often neglected because it is usually too much effort to detect individual orientations in sufficiently large number. The common measuring technique for determining ODFs are diffraction experiments (e.g. with X-rays) which, in a statistical form, yield the density distribution of crystallographic planes (poles) of a polycrystalline body. After measurement and data processing such data are usually plotted as iso-intensity lines of i) pole density in a stereographic projection, i.e. the so called pole figure (Wassermann and Grewen, 1962) or, after suitable mathematical transformation ("Pole figure inversion") (Bunge, 1969, Bunge, 1987) as ii) orientation density in a three-dimensional orientation space, (e.g. the Euler angle space). For the latter mostly sections through this space are given. Such representations can provide a good impression of the structure of the orientation distribution but for less experienced users these are usually too abstract and sometimes even confusing. Moreover, such representations in the form of "pictures" are rather voluminous and do not permit easy representation of the quantita- 
tive aspects of the structure and its changes with variation of experimental parameters.

These drawbacks often inhibit the application of textural methods in metallurgy and show the need for simpler and more comprehensive representations of orientation distributions which in an easy manner exhibit quantitatively the nature and intensity of their structural elements. In the literature two such concepts have been proposed and are already in use:

i) Description by means of ideal orientations

ii) Description by means of orientation tubes.

The first method is more useful for peak-type and the latter for axially-symmetric (fibre) type of textures. In cases where the texture is more complex both descriptions have been applied. In the present paper both methods will be checked for their validity and used in an extended and more quantitative form based on further exploitation of the three-dimensional orientation distribution function (ODF). Furthermore suitable plotting of texture data will be discussed.

\section{PEAK-TYPE TEXTURES}

The first approach when confronted with an unknown type of texture-in either pole figure or ODF form-should be to look for the positions and intensities of the main density maxima. In the case of a peak-type texture this method immediately gives a comprehensive (but still qualitative) idea of the main orientation concentrations. Figure 1 contains a list of the most frequently observed orientations in cubic metals with appropriate symbols which indicate their positions in the Euler space (Bunge's notation) in sections of constant $\varphi_{2}$. Additionally single letters as names are proposed, which could be used for an easy identification of these (and also of similar) orientations, in order to ease discussion and to avoid confusion.

As an example, Figure 2a shows the $\{111\}$ pole figure of an $\mathrm{Al}-0.007 \% \mathrm{Fe}$ recrystallization texture which consists mainly of two peak-type texture components. There are some shortcomings of the pole figure presentation which are already obvious even in this rather simple case. They have their origin in the use of a 


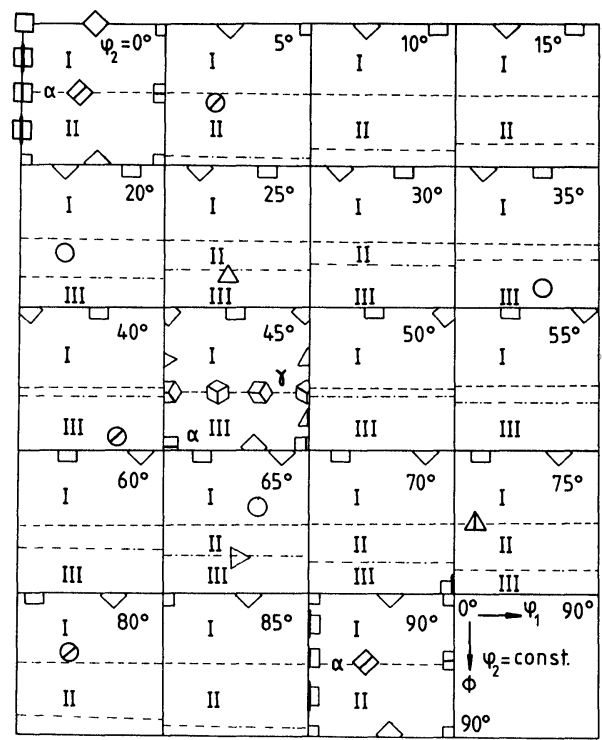

symbol- $\{$ hkl $\}\langle U v w\rangle$ - name

$\Delta-\{112\}\langle 111 \overline{1}\rangle-C$

$O-\{123\}\langle 634\rangle-$ Sor $R$

$\diamond-\{011\}\langle 21 \overline{1}\rangle-B$

$\square-\{011\}\langle 100\rangle-G$

$\Delta-\{255\}\langle 5 \overline{1} \overline{1}\rangle-\mathrm{TC}$

$\otimes-\{111\}\langle 11 \overline{2}\rangle-Y$

$\theta-\{111\}\langle 110\rangle-Z$

$\diamond-\{001\}\langle 110\rangle-$ rot.W

$\boxminus-\{011\}\langle 011\rangle-\operatorname{rot} G$

$\oslash-\{168\}\langle 21 \overline{1}\rangle-S / B$

(1) $-\{025\}\langle 100\rangle-W_{R D}$

$\square-\{001\}\langle 100\rangle-W$

$D-\{112\}\langle 110\rangle-A$

Figure 1 Some ideal orientations used for texture descritpion in cubic materials plotted in $\varphi_{2}=$ const. sections of the Euler space.

two-dimensional projection to portray three-dimensional data. In Figure $2 \mathrm{a}$ the four $\{111\}$ poles of the $\{001\}\langle 100\rangle$ cube orientation (squares) coincide with one pole of each of the four symmetrical equivalent $\{235\}\langle 83 \overline{5}\rangle \sim R$ orientations (one of these is indicated by filled circles). This deficiency can only be avoided by the additional measurement of different pole figures (e.g. $\{200\}$ ) or by the use of three-dimensional texture data (Figures $2 \mathrm{~b}, \mathrm{c}$ ). These are usually evaluated from several pole figures. Here directly the density $f(g)$ of each orientation $g$ is given in multiple of a random texture $(f(g)=1)$. Especially in the case of more complex textures the evaluation of three-dimensional orientation density data must be applied. Also characteristic peaks can be used to describe the texture. For example, in the texture of $95 \%$ cold rolled copper which has the appearance of a continuous orientation tube shown in Figures $3 a$ three main peaks (and two smaller ones) can be recognized in the ODF (Figure $3 b$ ) which determine the main features of the texture. 

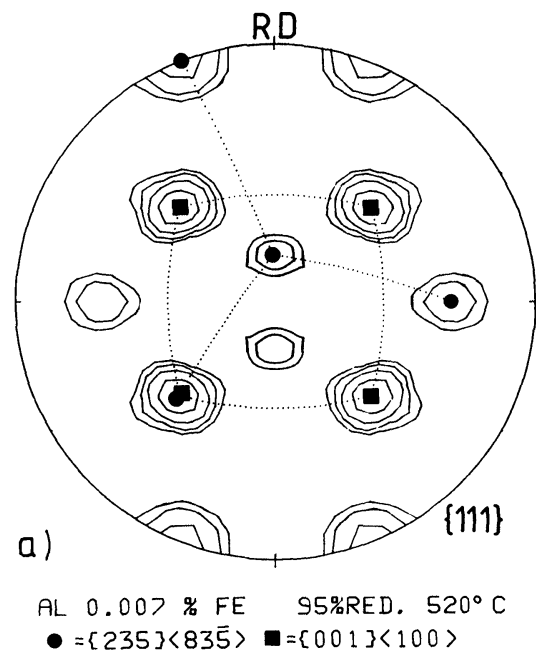

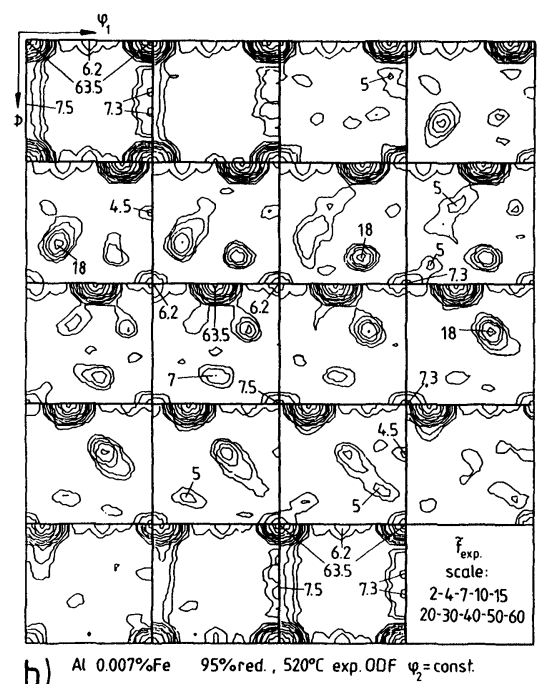

b) Al $0.007 \% \mathrm{Fe} \quad 95 \%$ red. , $520^{\circ} \mathrm{C}$ exp. ODF $\varphi_{2}=$ const.

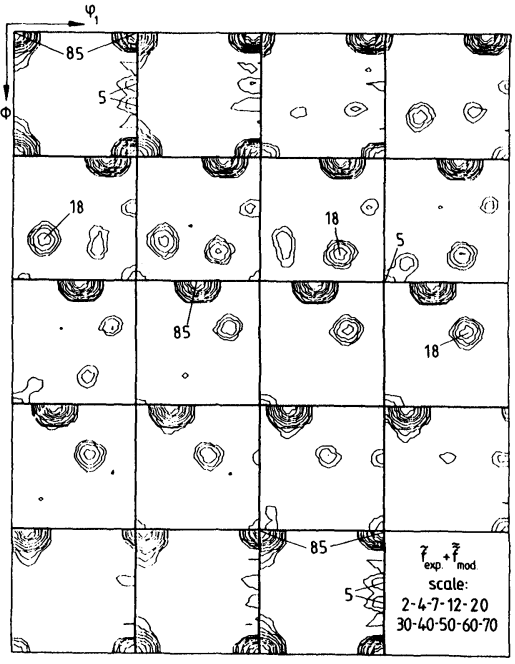

C) Al $0.007 \% \mathrm{Fe} \quad 95 \%$ red. $520^{\circ} \mathrm{Crecr}$. true ODF $\varphi_{2}=$ const.

Figure 2 Recrystallization texture of $\mathrm{Al}-0.007 \mathrm{w} \% \mathrm{Fe}$ cold rolled to $95 \%$ reduction and annealed $30 \mathrm{sec}$ at $520^{\circ} \mathrm{C}$ (Hirsch, Lücke, 1985). a) \{111\} pole figure. b) experimental (ghost carrying) ODF, c) Ghost corrected ODF. 


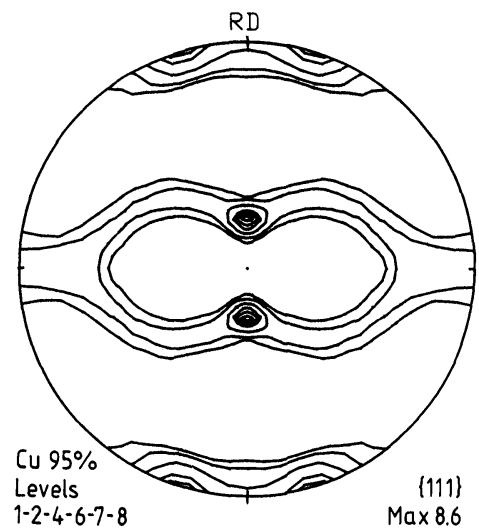

a)
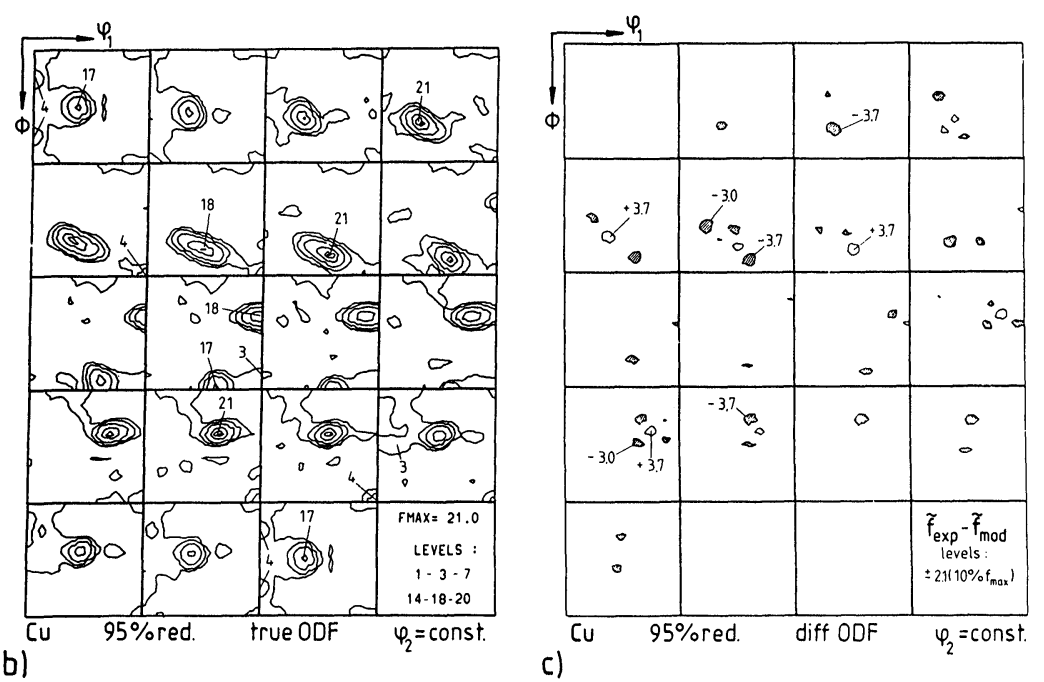

Figure 3 Rolling texture of pure copper (95\% reduction). a) $\{111\}$ pole figure, b) complete (ghost corrected) ODF, c) difference ODF (experiment minus model).

This method of considering peak intensities, however, is still only qualitative. To obtain the real intensities of the components requires also their scatterings to be taken into account, that means the intensities scattered around the central peaks must be integrated. This has been tried by using some arbitrarily fixed ranges in 
orientation space for integration (e.g. Kallend and Davies, 1972). A more useful and successfully applied method which involves the description of a texture peak by means of Gauss-type scattering functions avoids this ambiguity by individually determining the scatter width and thus the effective integration range for each textural component by means of a fitting procedure (Truszkowski, Pospiech, Jura, and Major, 1973). The intensity distribution $S$ around an orientation peak is then given as

$$
S(\omega)=S\left(\omega_{0}\right) \exp \left\{-\frac{\left(\omega-\omega_{0}\right)^{2}}{\psi^{2}}\right\}
$$

where $\omega$ describes the angular distance from the centre orientation $\omega_{0}$ around an arbitrary axis and $\psi$ the standard scatter (half) width of the Gauss distribution. Making use of this convention also different types of textures can quantitatively be compared since the scatter width is individually determined for each peak. In many cases, mainly in those involving isolated maxima, these yield a very good fit of experimental texture peaks (e.g. the $R$ component in Figure 2 (cf. Table 1a). But also characteristic scatterings of a peak can be constructed with the help of overlapping Gauss components, e.g. $W$ and $W_{R D}$ in Figure 1. Figure 3 even demonstrates the simulation of a more complex ODF of rolled pure copper by a model ODF which consists of four major and three minor Gausstype scattering components (Table 1b). Here the Gauss-model texture is also evaluated by the harmonic method (Truszkowski, Pospiech, Jura, Major, 1973) in form of series expansion coefficients $C_{1}^{\mu, v}(\mathrm{mod})$, as the experimental ones $C_{1}^{\mu \nu}(\exp )$ (Bunge, 1969) as:

$$
C_{1}^{\mu v}(\bmod )=\sum_{i=1}^{N} M_{i} \frac{\exp \left(-l^{2} \psi_{i}^{2} / 4\right)-\exp \left(1-(l+1)^{2} \psi_{i}^{2} / 4\right)}{1-\exp \left(-\psi_{i}^{2} / 4\right)} \dot{T}_{l}^{\mu v}\left(g_{i}\right)
$$

$N=$ number of Gauss model components

$l=$ series expansion degree, usually $l_{\max }=22$

$\dot{\sim}_{l}=$ generalized spherical harmonic functions.

The optimal fit of the experimental data can then easily be achieved by minimizing of the expression:

$$
\Delta X^{2}=\sum_{l, \mu, v} \frac{1}{2 l+1}\left(C_{l}^{\mu, v}(\exp )-C_{l}^{\mu, v}(\bmod )\right)^{2} \rightarrow \min
$$


Table 1 Some examples for results of quantitative texture analysis with the help of Gauss model components: $\{h k l\}\langle u v w\rangle$ are approximate Miller indices; $\varphi_{1}, \Phi, \varphi_{2}$ give the exact orientations, $M_{i}$ the volume fraction, $\psi_{0 i}$ the scatter (half) width of the best fitting Gauss model components

\begin{tabular}{llllllll} 
Name & $\sim\{h k l\}$ & $\langle u v w\rangle$ & $\varphi_{1}$ & $\Phi$ & $\varphi_{2}$ & $M_{i}[\%]$ & $\psi_{0 i}\left[^{\circ}\right]$ \\
\hline
\end{tabular}

a) $\mathrm{Al}-0.007 \% \mathrm{Fe}, 95 \%$ reduction, $520^{\circ} \mathrm{C}$ recrystallized (Figure 2 )

$\begin{array}{llllllrl}\sim W & 001 & 100 & 5^{\circ} & 0^{\circ} & 0^{\circ} & 32 & 5.5 \\ W_{\mathrm{RD}} & 015 & 100 & 4^{\circ} & 10^{\circ} & 0^{\circ} & 20 & 7.2 \\ W_{\mathrm{TD}} & 034 & 043 & 87^{\circ} & 36^{\circ} & 0^{\circ} & 3 & 5.3 \\ R & 235 & 835 & 60.2^{\circ} & 35^{\circ} & 55.9^{\circ} & 36 & 8.0 \\ \text { Phon } & - & - & - & - & - & 9 & -\end{array}$

b) $\mathrm{Cu} 95 \%$ reduction, as rolled (Figure 3 )

$\begin{array}{lllllrrr}C & 112 & 111 & 86^{\circ} & 31.5^{\circ} & 45^{\circ} & 27 & 7.2 \\ S & 123 & 634 & 61^{\circ} & 33.6^{\circ} & 63^{\circ} & 38 & 6.6 \\ B / S & 168 & 211 & 42^{\circ} & 39.2^{\circ} & 78^{\circ} & 18 & 5.6 \\ B & 011 & 211 & 33.5^{\circ} & 45^{\circ} & 0^{\circ} & 8 & 6.7 \\ B / G & 011 & 511 & 18^{\circ} & 42^{\circ} & 0^{\circ} & 3 & 6.2 \\ T C & 255 & 511 & 86^{\circ} & 82^{\circ} & 45^{\circ} & 3 & 6.6 \\ W_{\text {RD }} & 025 & 100 & 0^{\circ} & 21^{\circ} & 0^{\circ} & 3 & 6.0\end{array}$

c) $\mathrm{Al}$ alloy extruded (Figure 4)

$\begin{array}{llllllll}\langle 111\rangle \text { fibre } & h k l & 111 & \varphi_{1} & \Phi & \varphi_{2} & 75 & 7.2 \\ \langle 100<\text { fibre } & h k l & 100 & 0^{\circ} & \Phi & 0^{\circ} & 22 & 7.3 \\ \text { Phon } & - & - & - & - & - & 3 & \end{array}$

d) $\mathrm{Fe}$ (low carbon steel) $90 \%$ reduction, as rolled (Figures 5, 6)

\begin{tabular}{llllclrr} 
rot.W & 001 & 110 & $0^{\circ}$ & $0^{\circ}$ & $45^{\circ}$ & 12 & 11.2 \\
$A /$ rot.W & 115 & 110 & $0^{\circ}$ & $15^{\circ}$ & $45^{\circ}$ & 8 & 9.4 \\
$A$ & 112 & 110 & $0^{\circ}$ & $32.5^{\circ}$ & $45^{\circ}$ & 23 & 10.7 \\
$A_{\mathrm{RD}}$ & 557 & 110 & $0^{\circ}$ & $45^{\circ}$ & $45^{\circ}$ & 3 & 5.6 \\
$\sim A / Z$ & 6710 & 341 & $2.5^{\circ}$ & $42.5^{\circ}$ & $42.5^{\circ}$ & 28 & 9.8 \\
$W_{\text {ND }}$ & 100 & 520 & $0^{\circ}$ & $0^{\circ}$ & $22^{\circ}$ & 2 & 7.6 \\
$\gamma$ fibre & 111 & uvw & $\varphi_{1}$ & $55^{\circ}$ & $45^{\circ}$ & 24 & 9.8 \\
\hline
\end{tabular}

The quality of adaption can be checked by use of the value of $\Delta X^{2}$ or in more detail with the help of a "difference ODF" (Figure 3c), which is calculated by subtraction of the model from the experimental ODF. In the present case, the model ODF turns out to be impressively good and mostly difference values of $<10 \%$ occur.

This method of texture analysis involving model components leads to three very useful advances:

i) A quantitative, comprehensive description of the experimental 
texture is given which also contains information about the scatter associated with texture peaks. Integration of Eq. (1) gives the volume fractions, $M_{i}$, of each Gauss component $I$ :

$$
M_{i}=\frac{1}{2 \sqrt{ } \pi} Z_{i} S\left(\omega_{0}\right) \cdot \psi_{i}\left\{1-\exp \left(-\frac{\psi_{i}}{4}\right)^{2}\right\}
$$

where $Z_{i}$ is the multiplicity (symmetry) of the component $i$ in orientation space (which the model ODF automatically takes into account $)$. So the $C_{l}^{\mu, v}(\mathrm{mod})$ coefficients depend only on the orientation $\left(\varphi_{1}, \Phi, \varphi_{2}\right)_{i}$ and the values of $M_{i}$ and $\psi_{i}$ (Eq. (4)). For these the best fit values are calculated using a minimization procedure (Eq. 3). If a good approximation is achieved the whole texture is then concentrated in only few data (Table 1) which give a complete and quantitative representation of the texture, i.e., from these data the texture can be recalculated. These data are directly related to systematic texture changes and therefore provide a most convenient method of texture presentation and interpretation (Sec. 5). Since only information about the position and intensity of texture peaks is involved the whole procedure has been made fully automatic (Hirsch, Loeck, Loof, Lücke, 1984).

ii) In many cases the scattering of an experimental texture peak is non-isotropic, so that an isotropic Gauss-model component (Eq. (1)) does not provide an exact description. For such a component the minimization procedure calculates the mean scattering of the component, and the deviations from the Gauss shape then form peaks in the difference ODF (Figure 3c). These deviations, however, may also give useful information about the structure of a texture component by clearly demonstrating its scattering which otherwise is often very difficult to derive in the distorted geometry of orientation space. If these deviations are small enough, they can be neglected and the calculated volume fraction and scatter width in the Gauss-model analysis still provide adequate mean values. Sometimes, however, they are too large to be neglected or are of special interest in a more detailed texture analysis. In such cases the anisotropy may be described by placing the centre of a symmetrical peak somewhat apart from the symmetrical position or by the addition of auxiliary components (Hirsch, Lücke, 1987).

iii) Two serious deficiencies of the experimental ODF data can 
be eliminated by the use of model ODF calculations. These are the truncation error and the so-called "ghost" error. The truncation error in the series expansion method leads mainly to a systematic reduction in the intensity of sharp peaks. These, however, are relatively small if the degree of the series expansion $l_{\max }$ is high enough $\left(l_{\max }=22\right.$ is usually sufficient). But the ghost error strongly falsifies the ODF in a more undefinable way. The odd series expansion coefficients cannot directly be calculated from pole figure data and are therefore missing from the experimental ODF. This results in non-real (positive and negative) intensity peaks and systematic changes in the nature of the ODF plot especially at lower intensity levels which can be easily misinterpreted. These non-real peaks can also be observed in the Gauss model ODF if only even coefficients are used and therefore this method of model ODF calculation has been extended to derive the missing odd coefficients (Lücke, Pospiech, Virnich, Jura, 1981). (In Eq. (3) only even coefficients are involved, but odd ones can be calculated from Eq. (2).) In Figures $2 c$ and $3 b$ such a ghost corrected ODF is given which is "cleaned up" from most of the falsifications visible in the experimental ODF (Figure 2b), plotted with the same intensity levels.

\section{FIBRE-TYPE TEXTURES}

Some processes produce characteristic fibre type textures, containing a rather homogeneous orientation distribution which can be described by a rotation of orientations around a common fibre axis. This occurs in ideal form after axi-symmetric deformation (e.g. wire drawing or extrusion (Figure 4a). For these cases usually a presentation of the orientation distribution by plotting the density distribution of the principle axis in the reference frame of the crystal coordinate system, the so-called "inverse pole figure" is chosen, e.g. the extrusion direction ED density plotted in the basic triangle of the stereographic projection (Figure $4 \mathrm{~b}$ ).

However, also here a comparison of different samples might be difficult by plotting only these figures. A method which allows to detect slight variations in fibre textures is to plot the integral pole figure density data for each pole circle around the central fibre axis 

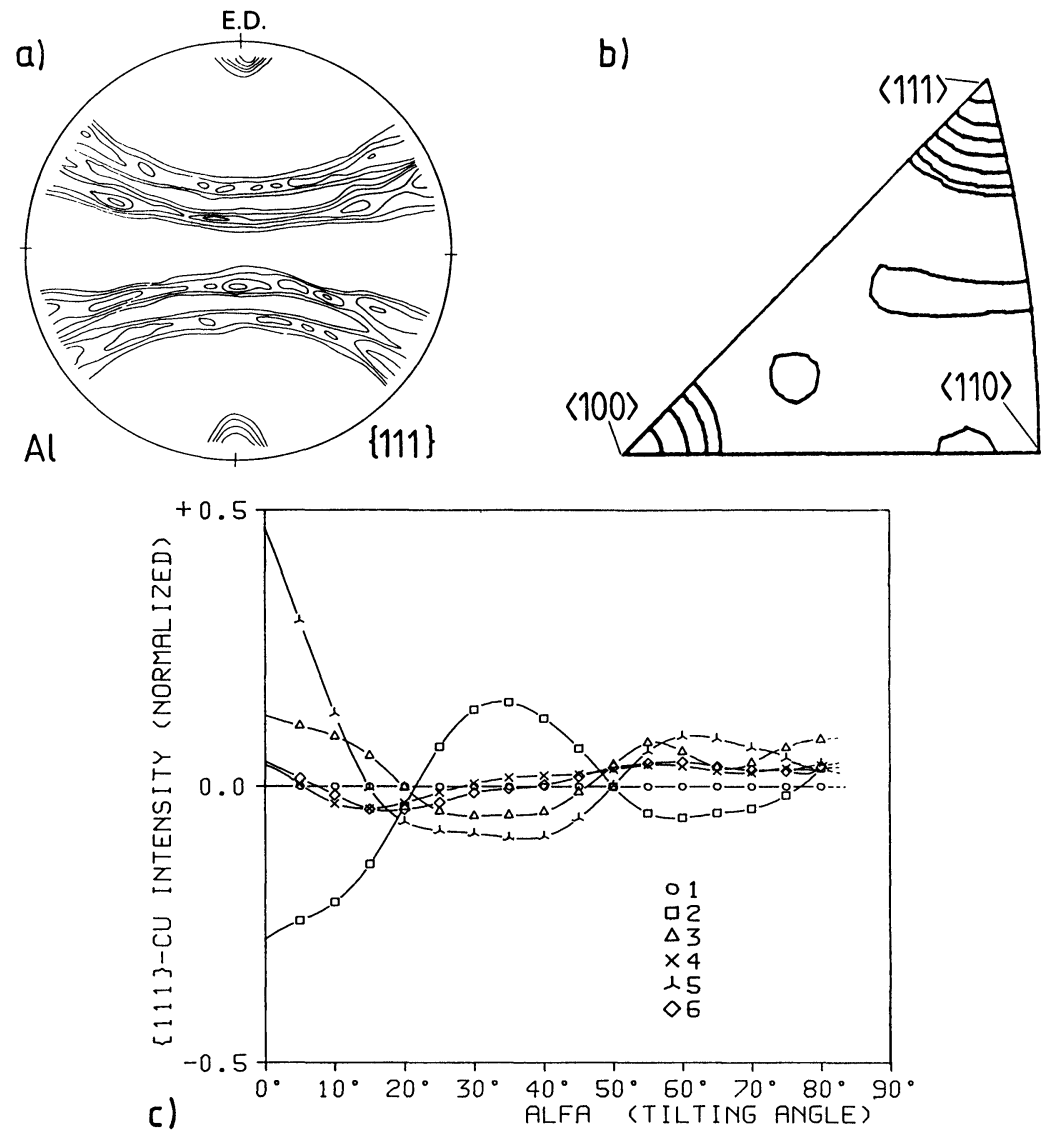

Figure 4 Texture of an extruded $\mathrm{Al}$ wire. a) $\{111\}$ pole figure (E.D. = Extrusion Direction), b) inverse pole figure of the extrusion direction, c) analysis of slight variations of different fibre textures with the help of plotting the mean pole circle intensity as function of the angular distance $\alpha$ to the fibre axis, normalized to a reference texture.

(e.g. ND with $\beta=0^{\circ}-360^{\circ}$ ) as function of the tilting angle $\alpha$. If their intensity data $I_{i}(\alpha)$ are furthermore normalized to a reference texture $I_{R}(\alpha)$ by $I_{N}(\alpha)=\left(I_{i}(\alpha)-I_{R}(\alpha)\right) / I_{R}(\alpha)$ a rather clear comparison and the analysis of slight variations of similar fibre textures is possible.

An example is given in Figure $4 \mathrm{c}$ where the $\{111\}$ intensity for 
different samples (2-6) normalized to that of sample 1 is plotted versus $\alpha$ showing characteristic differences in the fibre texture intensity of samples 2 and 5 .

In case of ideal fibre shape the orientation distributions can be simulated using fibre type components with constant intensity in one direction and Gauss type scattering in the others. These can also be

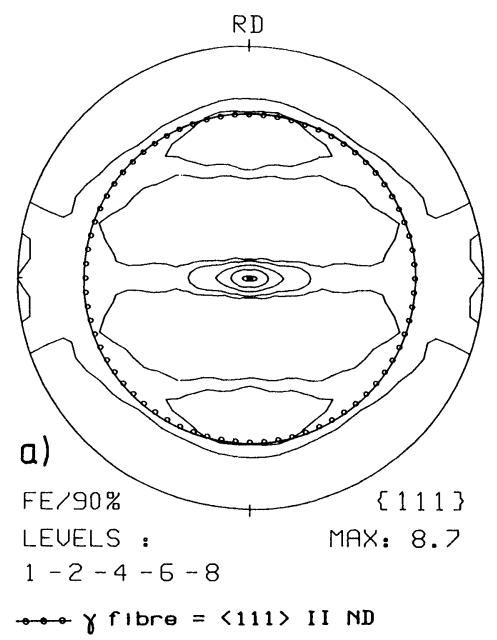

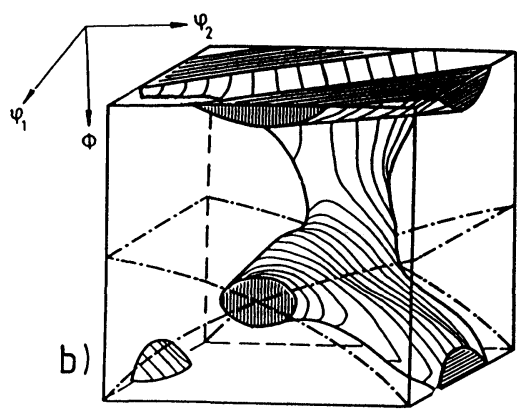

ODF Fe $90 \%$ red.

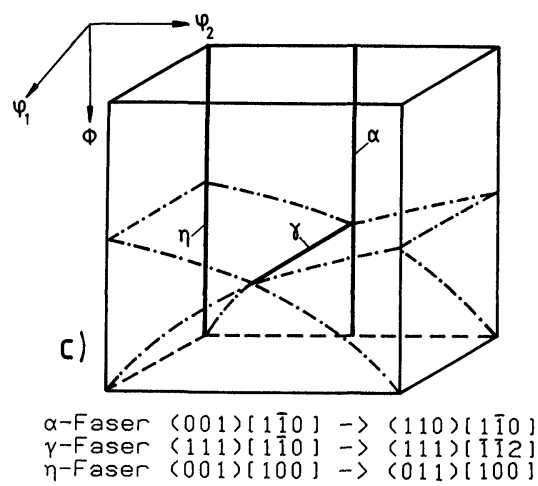

Figure 5 Rolling texture of Fe (low carbon steel) cold rolled to $90 \%$ reduction. a) $\{111\}$ pole figure, b) $3 \mathrm{D}$ plot in Euler space, c) Schematic plot of principle fibres of bcc rolling textures in the Euler space. 
given in the form of series expansion coefficients (Jura, 1988):

$$
\begin{aligned}
C_{l}^{\mu s}(\mathrm{mod})= & \frac{4}{\sqrt{ } 2 \pi} M_{i}\left[\frac{1}{\operatorname{erf}\left(\psi_{i} / 2\right)} \sum_{n=1}^{l_{\max }} \varepsilon_{n} \cdot m_{l}^{n} \exp \left(\frac{\left.1-n^{2}\right) \psi_{i}^{2}}{4}\right)\right] \\
& \times \dot{k}_{l}^{\mu}\left(h_{i}\right) \cdot k_{l}^{s}\left(y_{j}\right)
\end{aligned}
$$

Here $h_{i}$ and $y_{i}$ give the orientation (spherical coordinates) of the fibre in the crystal and specimen reference frame, repectively. The $M_{i}$ and $\psi_{i}$ are the volume fraction and scatter width of the fibre component with $\varepsilon_{n}=1(n=0)$ or $=2(n \neq 0)$ and the $m_{l}^{n}$ defined by equation:

$$
\cos n \psi=\sum_{1=0(2)}^{n} m_{l}^{n} \cdot P_{l}(\cos \psi) .
$$

Making use of the same procedure as described above (Sec. 2) for the adaption of $C_{l}^{\mu \nu}(\mathrm{mod})$ coefficients to the experimental ones a quantitative description in form of volume fractions and scatter widths can be achieved (Table 1c).

Also for the case of the rolling and recrystallization textures of bcc metals a description based on the use of orientation fibres can be very useful. Here, a concentration of orientations with a
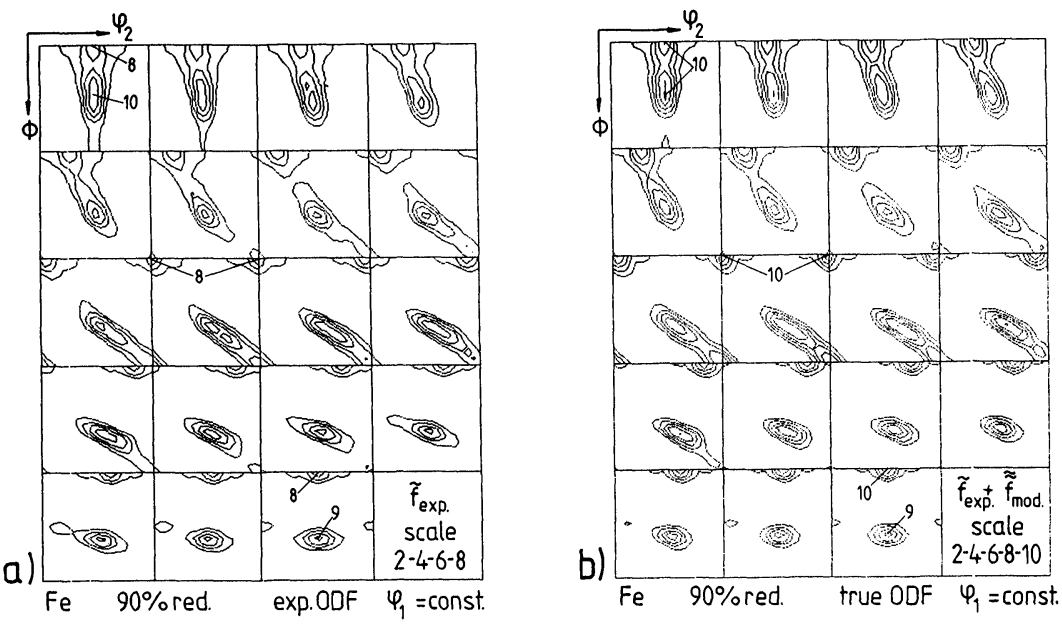

Figure 6 Rolling texture of Fe cold rolled to $90 \%$ reduction ( $\triangle$ Figure $5 \mathrm{a}, \mathrm{b}$ ), plotted in sections of $\varphi_{1}=$ constant. a) experimental (ghost carrying) ODF, b) true (ghost corrected) ODF. 
common $\langle 111\rangle$ sheet normal (the $\gamma$ fibre) or a common $\langle 110\rangle$ rolling direction (the $\alpha$ fibre) is often observed (Lücke, Därmann, Hirsch, 1985). Figures 5 and 6 show an example of the texture of a rolled low carbon steel which contains a strong and almost ideal $\langle 111\rangle \gamma$ fibre. With the help of ODF model calculations (Table 1d) the experimental ODF (Figure 6a) can be cleaned up from ghost errors (Figure 6b). In comparison to the peak-type textures (Figures 2,3 ), however, the effect of ghost correction is much less. (Mainly the tail of the $\alpha$ fibre in the $\varphi_{1}=0^{\circ}$ section is somewhat shortened.) This is due to the fact that complete fibres with symmetrical fibre axis (e.g. $\langle 100\rangle$ or $\langle 111\rangle$ ) are centrosymmetric and therefore completely described by even series expansion coefficients only (Jura, 1988).

\section{MIXED TYPE TEXTURES}

In some textures fibres are not present in their complete form, but restricted to limited rotation angles. For example in bcc rolling textures the $\alpha$ fibre in the $\varphi_{1}=0^{\circ}$ section at $\varphi_{2}=45^{\circ}$ reaches only up to $\Phi \approx 55^{\circ}$ (Figure 5). In such cases either a fibre-type component as given by Eq. (5) but reduced by a negative Gauss-type component (Eq. (2)) can be used to give an approximate (quantitative) description or a number of overlapping Gauss components (Table 1d), which have the advantage to include all observed characteristic density variations along the fibre.

Also fcc rolling textures can have the appearance of tube like textures. In Figure 7 the tube character of a $90 \%$ rolled $\mathrm{Cu} 2.5 \% \mathrm{Zn}$ alloy with a copper-type rolling textures is demonstrated by plotting a single contour level of the ODF in the three-dimensional Euler angle space. In order to avoid overlapping with the symmetric equivalent tubes these (and some minor peaks) have been omitted. Their occurrence in the other two (symmetrically equivalent) subspaces II and III (Hansen, Pospiech, Lücke, 1978) are plotted in Figure $7 \mathrm{a}$ in the adjacent cell of the Euler space which usually is given as $0^{\circ} \leqq \varphi_{1}, \Phi, \psi_{2} \leqq 90^{\circ}$ (e.g. Figures $2 \mathrm{~b}, 3,5$ ). Figure $7 \mathrm{a}$ clearly shows the two characteristic fibres, called $\alpha$ and $\beta$ of fcc rolling textures (Hirsch, Lücke, 1988), plotted schematically in Figure $7 b$. 


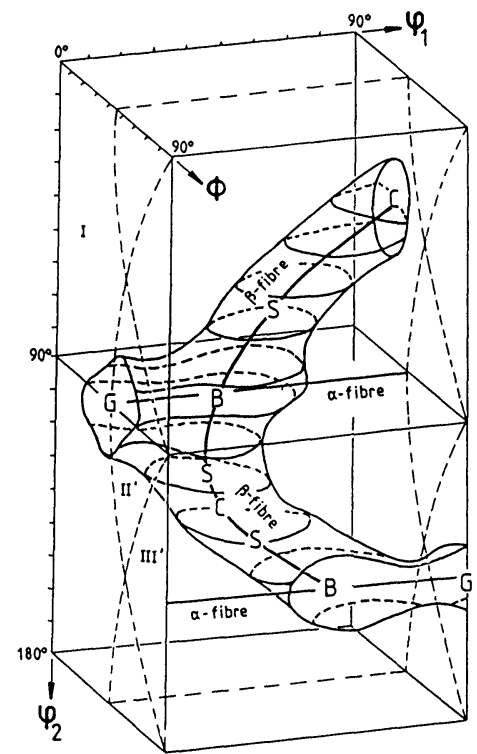

a)

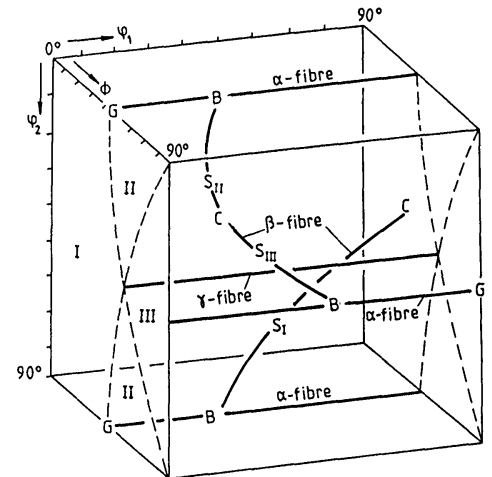

b)

Figure $73 \mathrm{D}$ plot of a typical fcc rolling texture in Euler angle space. To avoid overlapping the occurrence of the experimental texture tube in the three subspaces (I, II, III) is plotted in two adjacent cells. Symmetrically equivalent tubes and minor peaks are omitted. The main texture components and fibres are indicated. a) Experimental texture in $\mathrm{Cu} 2.5 \% \mathrm{Zn}, 90 \%$ reduction, b) Schematic plot of fcc rolling texture fibres.

In two-dimensional projections of the ODF, e.g. pole figures, the fibres and also other minor peaks may easily overlap. By omitting some of these, that means by making a selective projection of only one feature and only of one of its symmetrically equivalent variants, however, this confusing effect can be removed. The rolling texture tube given in Figure 7 projected along $\varphi_{2}$ results in a $\{100\}$ pole figure or projected along $\varphi_{1}$, in an inverse pole figure of the sheet plane normal (Hansen, Poepiech, Lücke, 1978). As the third possible case in Figure $8 \mathrm{~b}$ the (selective) projection along $\Phi$ is given for a $95 \%$ rolled $\mathrm{Cu} 5 \% \mathrm{Zn}$ alloy. As can be noticed from Figure 7 in this case $\varphi_{2}$ varies from $45^{\circ}$ to $135^{\circ}$ and $\varphi_{1}$, from $0^{\circ}$ to $90^{\circ}$. The orientations occurring in this figure and the variation of $\Phi$ are schematically plotted in Figure $8 \mathrm{a}$. In this projection the two 


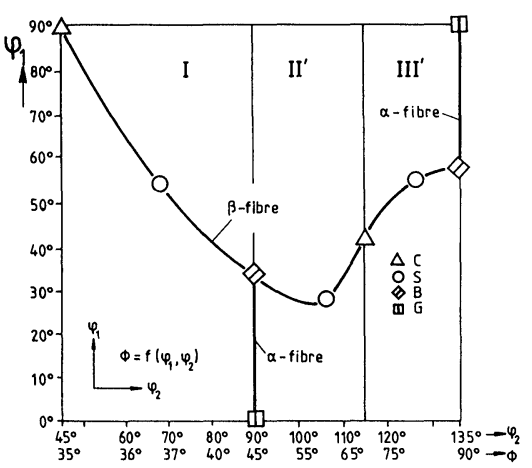

a)

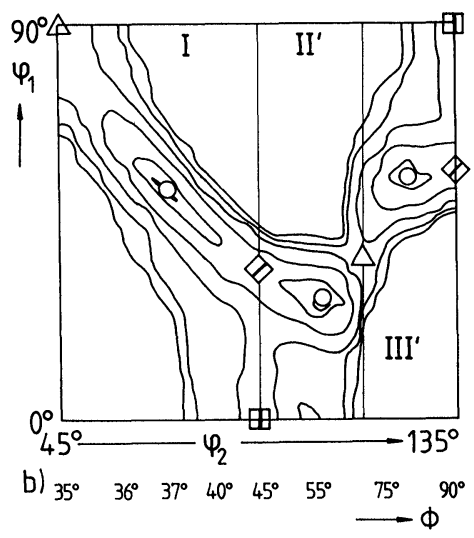

Figure $8 \Phi$-projection of the copper type rolling texture. a) Schematic plot of the occurring main texture components and fibres, b) $\Phi$-projection of a Cu5\% $\mathrm{Zn}$ rolling texture ( $95 \%$ reductions).

characteristic fibres $(\alpha$ and $\beta$ ) occurring in fcc rolling textures can be seen separately and the transition of the copper-type ( $\alpha$ and mainly $\beta$ fibre) to the brass-type rolling texture (only $\alpha$ fibre with a peak at $B\{011\}\langle 211\rangle$, cf. Sec. 5) and the corresponding changes from a fibre-type to a peak-type texture can be analyzed (Hirsch, Lücke, 1988).

Also for the description and interpretation of rolled fcc materials, fibre-type textures have been proposed. A first description of this type was given by Grewen and Wassermann (Grewen, Wassermann, 1955). In order to include the systematic scatterings between the peaks in $\{111\}$ and $\{200\}$ pole figures of the fcc rolling texture they mainly applied limited fibres around certain $\langle 111\rangle$ poles of the two main components $C$ and $B$. These approximately describe the form of the typical appearance of the copper-type rolling texture as observed in a $\{111\}$ pole figure (Figure 10a). Later Lücke et al. (Lücke, Därmann, Hirsch, 1985; Hirsch, Lücke, 1988) made use of $\langle 110\rangle$ fibres which are less evident since $\{110\}$ pole figures are seldom presented (Figure 10d). One of these consists of orientations with a common $\langle 110\rangle$ axis parallel to the sheet normal (called $\alpha$ fibre) while the other consists of orientations with a common $\langle 110\rangle$ axis tilted about $60^{\circ}$ from the normal direction towards the rolling direction (called $\beta$ fibre). The latter is often referred to in the literature as the "skeleton line" of fcc rolling textures. (It was first 

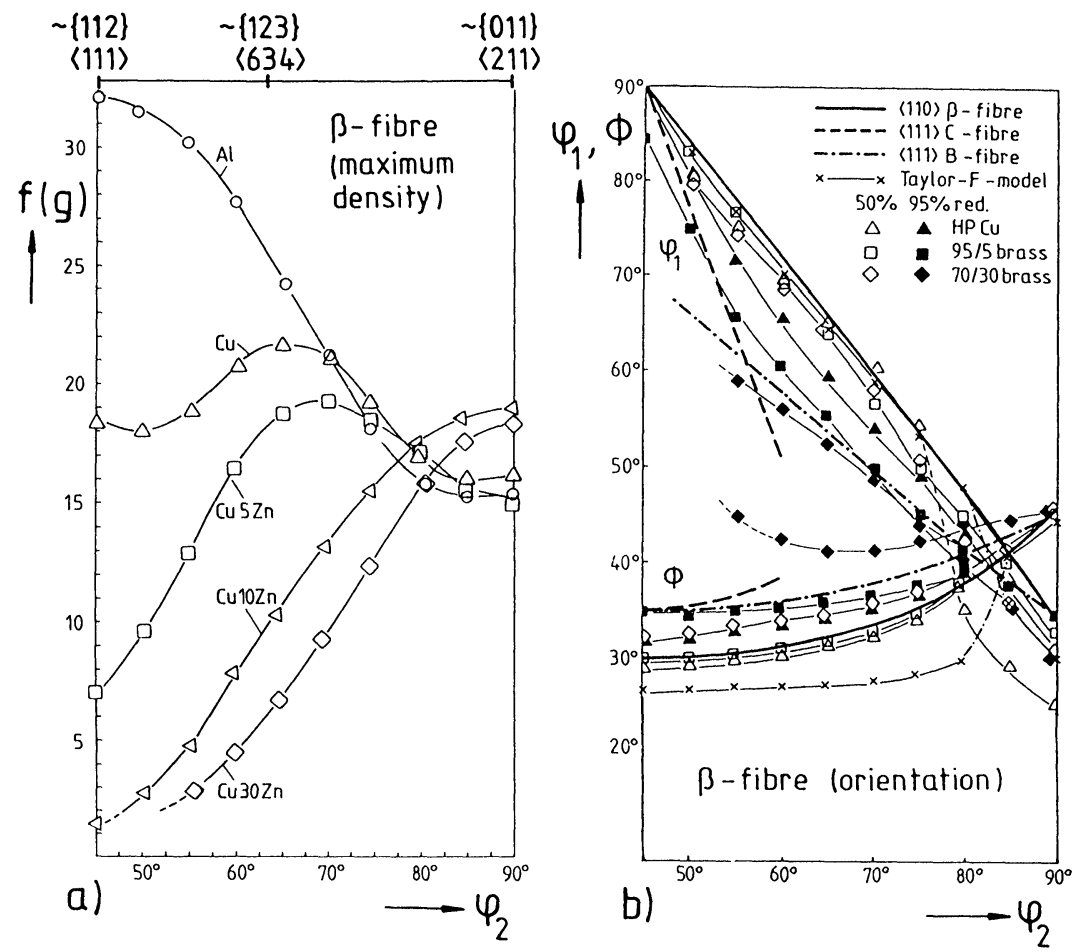

Figure $9 \beta$ rolling texture fibre in different fcc materials (Hirsch, Lücke, 1988). a) maximum orientation density $f(g)$ vs. $\varphi_{2}$ along the $\beta$ fibre (skeleton line), b) exact position of the $\beta$ fibre, $\varphi_{1}$ and $\Delta$ vs. $\varphi_{2}$, and comparison to descriptive and theoretically predicted texture fibres.

introduced by Bunge and Tobisch (1968) as the line of maximum orientation density running through the orientation space but without indicating a specific fibre axis.) As can be seen in Figures $10 \mathrm{~b}$ and $\mathrm{d}$ the $\langle 110\rangle \beta$ fibre gives a much better description of the experimentally observed fibre position than the $\langle 111\rangle$ fibres (Figures 10a,c) proposed by Grewen and Wassermann, 1955.

While for bcc metals a rather constant orientation density along the $\gamma$ fibre is often observed, in fcc metals at certain positions of the $\beta$ fibre already at medium strains characteristic concentrations occur. With increasing strain the fibre-type texture changes continuously into a peak-type texture and for high and medium SFE $(\mathrm{Al}$ and $\mathrm{Cu})$ often a mixed texture type occurs above a certain 

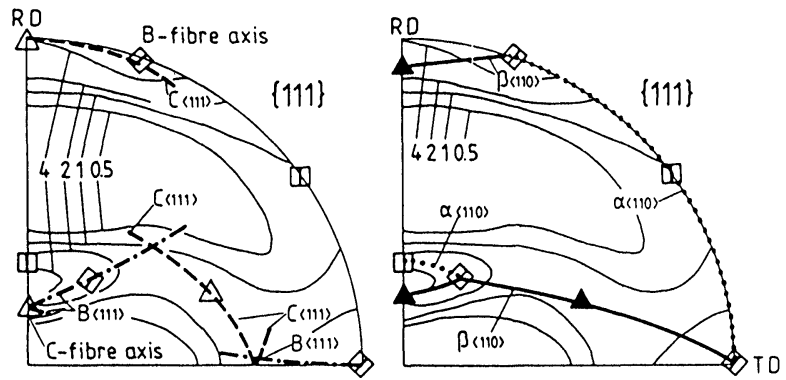

a) $\mathrm{HPCU} 75 \%$ red. Max. $=6.0$

- - $[(111)$-fibre $\Delta\{112\}\langle 111\rangle$

-D B (111)-fibre $\otimes(011)\langle 211\rangle$

(1) $(011)(100)$
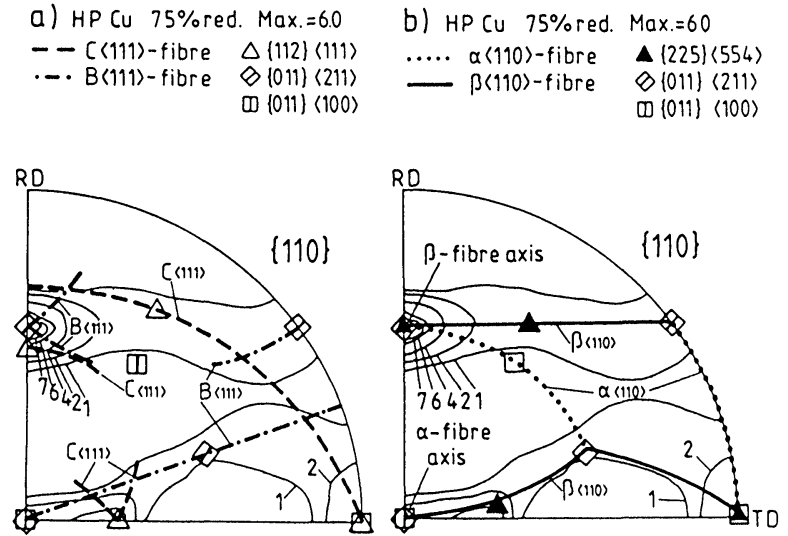

C) HPCu $95 \%$ red. Max. $=8.3$

d) $\mathrm{HPCu} 95 \% \mathrm{red}$. Max. $=8.3$

- - $\{(111)-$-fibre $\quad \Delta(112\}(111)$

- B B (111)-fibre $\diamond(011\}\langle 211)$

$\begin{array}{ll}\text {.... } \alpha(110) \text {-fibre } & \Delta(225)(554) \\ -\alpha\langle 110\rangle \text {-fibre } & \diamond(011)(211)\end{array}$

(1011\} (100)

ه (011) (100)

Figure 10 Descriptive texture fibres for copper type rolling textures and comparison to experimental pole figures of rolled pure copper. a) and b) $\{111\}$ pole figure of $75 \%$ rolled $\mathrm{Cu}, \mathrm{c})$ and d) $\{110\}$ pole figure of $95 \%$ rolled $\mathrm{Cu}$.

strain level (which then can better be described by overlapping peak-type texture components, cf. Sec. 2, Figure 3 and Table 1b). This is demonstrated in Figures 9a, where the maximum orientation densities $f(g)$ long the $\beta$ fibres are plotted for different fcc metals, all at $95 \%$ rolling reduction. Depending on the stacking fault energy (SFE) characteristic peaks develop for the different materials.

The $\alpha$ fibre in Euler space (Figure 7) runs from the " $G$ " orientation through " $B$ " to "rotated $G$ " (cf. Figure 1) and is defined by the variation of the angle $\varphi_{1}$ at constant $\Phi$ and $\varphi_{2}$. The 
position of this fibre is usually stable in various textures. The $\beta$ fibre runs in Euler space from about the " $C$ " orientation through " $S$ " (cf. Figure 1, Table 1) and meets the $\alpha$ fibre at about the " $B$ " orientation. It slightly varies in its exact orientation course depending on the composition and SFE of the material and the degree of rolling (Hirsch, Lücke, 1988). The exact position of the $\beta$ fibre for some fcc materials after $50 \%$ and $95 \%$ rolling reduction is plotted as a projection of the $\beta$ fibre on the $\Phi=0^{\circ}$ and on the $\varphi_{1}=0^{\circ}$ plane (i.e. $\Phi$ and $\varphi_{1}$ plotted versus $\varphi_{2}$ ) in one figure (Figure $9 \mathrm{~b}$ ). Also the descriptive fibres described above (Figure 10) are inserted in Figure 9b.

In Figure 9b, where all overlapping effects are avoided, it can easily be noticed that for high and medium SFE ( $\mathrm{Cu}$ and $\mathrm{Al}$ )- and especially at low reductions where the fibre character is most pronounced-the $\langle 110\rangle \beta$ fibre is the best description for the course of the experimentally observed $\beta$ fibres. For low SFE material (e.g. $70 / 30$ brass) the $\langle 111\rangle \beta$ fibre (but not the $\langle 111\rangle C$ fibre!) is in good agreement at high strain. But at that stage the peak character of the texture predominates.

\section{EXAMPLES OF TEXTURE ANALYSIS}

Texture development can be well illustrated by using the methods described above. Both the description by peak- and fibre-type components can be considered for the cases of rolling textures in fcc metals with different SFE (Hirsch, Lücke, 1988).

In Figure 11a the volume fractions $M_{i}$ of the main rolling texture components of high purity copper are plotted as a function of rolling strain $\varepsilon$. Even though the orientation distribution at low reductions $(\varepsilon \leqq 1)$ has many of the characteristics of a texture fibre (see Sec. 3) the method of fitting by isotropic Gauss components can be applied. It is then necessary to introduce also a so-called background component "Phon" which describes the random part of the texture. This component, which in case of a completely random orientation distribution before rolling would be $100 \%$, decreases constantly with increasing strain as the main rolling texture components develop: these are the $C, S$, and $B$ orientation (cf. Figure $1)$. The $G$ orientation is present in the early stages of texture 

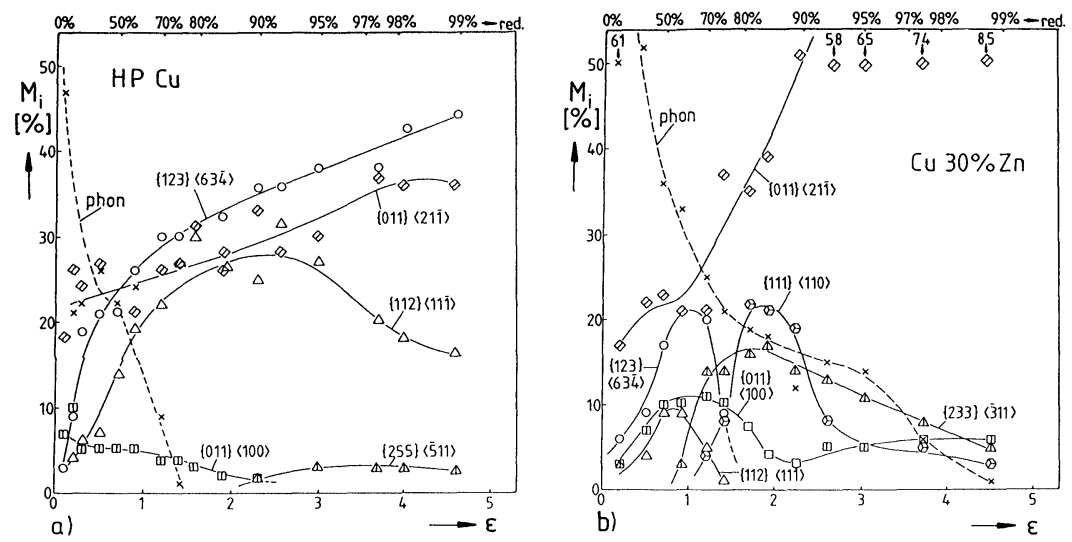

Figure 11 Volume fractions $M_{i}$ of rolling texture components as derived from Gauss model analysis plotted against rolling strain $\varepsilon$ (Hirsch, Lücke, 1988). a) pure copper, b) $70 / 30$ brass.

development but decreases continuously with increasing strain. The main components all steadily increase, except the $C$ orientation, which reaches a maximum when at very large strains $(\varepsilon \geqq 3)$ its twin appears.

In the case of $70 / 30$ brass (Figure 11b) the texture development at very low strains is similar to that in pure copper but then changes drastically. The maximum for the $C$ orientation is reached at moderate strains $(\varepsilon<1)$ and the strength of the $S$ component also decreases. These decreases are accompanied by the appearance of components with twin and rotated twin orientations that are widely

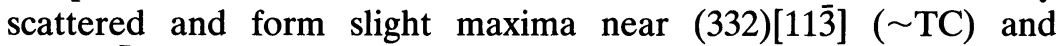
(111)[110] ("Y"). These orientations are also unstable and finally disappear with further defomation whereas the $G$ orientation and particularly the stable $B$ orientation strongly increase in strength.

The precise position of the $\beta$ fibre also varies systematically with strain and SFE (Figure 9b). At low strain and high SFE it is very close to the line predicted theoretically by the Taylor theory of polycrystalline plasticity but with increasing strain it shifts systematically to smaller $\varphi_{1}$ and higher $\Phi$ values which can be understood in terms of the "relaxed constraints theory" (Hirsch, Lücke, 1988).

In pure copper the $\beta$ fibre develops homogeneously with increasing strain although systematic peaks are formed at the three main rolling texture components $(C, S, B$, Figure 9a). At very high 
strains the peak in the middle of the $\beta$ fibre at the $S$ orientation predominates.

At medium strains the $\alpha$ fibre already begins to deteriorate and a strong peak, which is also part of the more steadily increasing $\beta$ fibre, remains at the $B$ orientation. In $70 / 30$ brass the orientations along the $\beta$ fibre also strongly concentrate near the $B$ orientation (Figure 9a) so that at high strains this texture consists principally of the $B$ component with some scattering along the two fibres. It is generally accepted, that the formation of mechanical twins is responsible for these strong texture changes observed in fcc metals of low stacking fault energy (e.g. 70/30 brass), as first pointed out by Wassermann (Wassermann, 1963). The complete process of texture transition from the copper-type to the brass-type rolling texture, however, is much more complicated and has recently been analyzed in detail with the help of ODF analysis described above (Hirsch, Lücke, 1988).

\section{References}

Bunge, H. J. (1969). Mathematische Methoden der Texuranalyse, Akademie-Verlag Berlin.

Bunge, H. J. and Tobisch, J. (1968) Z. Metallkde. 59, 471-475.

Bunge, H. J. (ed.) (1987). Theoretical Methods of Texture Analysis, DGM-Verlag, Oberursel 1987.

Grewen, J. and Wassermann, G. (1955). Acta Metall. 3, 354-360

Hansen, J., Pospiech, J. and Lücke, K. (1978). Tables for Texture Analysis of Cubic Crystals, Springer-Verlag, Berlin.

Hirsch, J., Loeck, M., Loof, L., and Lücke, K. (1984). Proc. ICOTOM 7, Noordwijkerhout 765-770.

Hirsch, J. and Lücke, K. (1985). Acta Metall. 33, 1927-1938.

Hirsch, J. and Lücke, K. (1987). Theoretical Methods of Texture Analysis, ed. by H. J. Bunge, DGM-Verlag, Oberursel, 53-62.

Hirsch, J. and Lücke, K. (1988). Acta Metall. in press.

Hirsch, J. (1984). Doctoral thesis, RWTH Aachen.

Jura, J. (1988). Bul. Pol. Sci. and Proc. ICOTOM 8, Santa Fe, in press.

Kallend, J. S. and Davies, G. J. (1972). Texture 1, 51-69.

Lücke, K., Pospiech, J., Virnich, K. H., and Jura, J. (1981). Acta Metall. 29, 167-185.

Lücke, K. (1981). Proc. ICOTOM 6, Tokyo 14-38.

Lücke, K., Därmann, C., and Hirsch, J. (1985). Trans. Ind. Inst. Met., 38, 6 496-509.

Truszkowski, W., Pospiech, J., Jura, J., and Major, B. (1973). proc. "3ème colloque européen sur les textures ..." Pont-à-Mousson 235-257.

Wassermann, G. and Grewen, J. (1962). Texturen metallischer Werkstoffe, SpringerVerlag, Berlin-Göttingen-Heidelberg.

Wassermann, G. (1963). Z. Metallkde. 54, 61-65. 